SWAT/02/359

December 2002

\title{
Mass Generation without Phase Coherence at Nonzero Temperature
}

\author{
Costas G. Strouthos and David N. Walters \\ Department of Physics, University of Wales Swansea, \\ Singleton Park, Swansea SA2 8PP, U.K.
}

\begin{abstract}
We present results from numerical simulations of the $2+1 d S U(2) \otimes S U(2)$ Nambu-Jona-Lasinio model with $N_{f}=4$ fermion flavors at zero and nonzero temperature $T$. At zero temperature, critical exponents extracted from the scaling of the order parameter and fermion mass are found to be consistent with next-to-leading order predictions of the $1 / N_{f}$ expansion. At nonzero temperature we observe fermion mass generation despite the lack of chiral symmetry breaking, which is forbidden by the Colemann-Mermin-Wagner theorem for all $T>0$. We study the effects of lattice discretisation and finite volume on the dynamically generated fermion mass. By studying the lattice dispersion relation we also show that in the hot phase there is no significant temperature induced modification to the speed of light. Studies of the equation of state are made by measuring the pressure as a function of temperature and comparison is made with large- $N_{f}$ predictions.
\end{abstract}




\section{Introduction}

The nature of QCD and related field theories at finite temperature remains a highly active area of both analytic and numerical research. By studying the high temperature transition in strongly interacting theories we gain insight not only into the hot chirally symmetric phase, but also the cold phase, in which spontaneous chiral symmetry breaking leads to a dynamically generated fermion mass.

In $2+1$ dimensional field theories the nature of the transition can be particularly interesting. It has been shown [2, 3] that in the three-dimensional Gross-Neveu model with a $U(1)$ chiral symmetry at nonzero temperature, there is a regime at temperatures above the Berezinzkii-Kosterlitz-Thouless transition in which the fermions acquire nonzero mass despite the absence of phase coherence. An analogous phenomenon is found in the physics of strong coupling or low carrier density superconductors. In these materials Cooper-pair formation occurs below a temperature $T=T^{*}$ whilst the $U(1)$ symmetry remains manifest. The "local" gap modulus which is neutral under $U(1)$ rotations is nonzero, whilst the phase of the gap fluctuates violently [1]; this is known as the pseudogap phase. It is only below a temperature $T=T_{c} \ll T^{*}$ that phase fluctuations cease and the material enters a true superconducting phase. This separation of the temperatures of pair formation and pair condensation has been known of for many years. It remains an open question, however, whether the dynamic generation of fermion masses without the breaking of chiral symmetry could be phenomenologically relevant in particle physics.

In this paper we present a thermodynamic study of the $2+1 d S U(2) \otimes S U(2)$ Nambu-Jona-Lasinio (NJL) model on the lattice, with the aim of showing that these phenomena can occur in theories with more phenomenologically motivated symmetries.

In the continuum, the model is described by the Euclidean Lagrangian density

$$
\mathcal{L}=\bar{\psi}_{i} \not \partial \psi_{i}-\frac{g^{2}}{2 N_{f}}\left[\left(\bar{\psi}_{i} \psi_{i}\right)^{2}-\left(\bar{\psi}_{i} \gamma_{5} \vec{\tau} \psi_{i}\right)^{2}\right],
$$

where $\vec{\tau} \equiv\left(\tau_{1}, \tau_{2}, \tau_{3}\right)$ are the Pauli spin matrices which run over the internal $S U(2)$ isospin symmetry and are normalised such that $\operatorname{tr}\left(\tau_{\alpha} \tau_{\beta}\right)=2 \delta_{\alpha \beta}$. The index $i$ runs over $N_{f}$ fermion flavors and $g^{2}$ is the coupling constant of the four-fermi interaction.

The model is chirally symmetric under $S U(2)_{L} \otimes S U(2)_{R} ; \psi \rightarrow\left(P_{L} U+P_{R} V\right) \psi$, where $U$ and $V$ are independent global $S U(2)$ rotations and the operators $P_{L, R} \equiv \frac{1}{2}\left(1 \pm \gamma_{5}\right)$ project onto left and right handed spinors respectively. It is also invariant under $U(1)_{V}$ corresponding to a conserved baryon number.

The theory becomes considerably easier to treat, both analytically and numerically, if we introduce scalar and pseudo-scalar fields denoted by $\sigma$ and $\vec{\pi}$ respectively. The bosonised Lagrangian is

$$
\begin{aligned}
\mathcal{L} & =\bar{\psi}_{i}\left(\not \partial+\sigma+i \gamma_{5} \vec{\pi} \cdot \vec{\tau}\right) \psi_{i}+\frac{N_{f}}{2 g^{2}}\left(\sigma^{2}+\vec{\pi} \cdot \vec{\pi}\right) \\
& =\bar{\psi}_{i}\left(\not \partial+\sigma+i \gamma_{5} \vec{\pi} \cdot \vec{\tau}\right) \psi_{i}+\frac{N_{f}}{4 g^{2}} \operatorname{tr} \Phi^{\dagger} \Phi
\end{aligned}
$$


where the combination $\Phi \equiv \sigma+i \vec{\pi} \cdot \vec{\tau}$ is proportional to an element in the chiral group such that the model is invariant under the rotation $\Phi \rightarrow V \Phi U^{-1}$.

Apart from any obvious numerical advantages this relatively simple model has various interesting properties:

(i) the spectrum of excitations contains distinguishable baryons and mesons, i.e. the elementary fermions $q$ and the composite $q \bar{q}$ states;

(ii) at zero temperature and sufficiently strong coupling $g^{2}>g_{c}^{2}$, chiral symmetry is spontaneously broken leading to a dynamically generated fermion mass equal to $M(0)=\langle\sigma\rangle$ in the large- $N_{f}$ limit; the pion fields are the associated Goldstone bosons;

(iii) for dimensionality $2<d<4$ there is an interacting continuum limit at a critical coupling, which for $d=3$ has a numerical value $g_{c}^{2} / a \approx 1.0$ to leading order in $1 / N_{f}$ with a lattice regularisation employed;

(iv) the global symmetries are the same as those of 2 flavor QCD, for which reason its four-dimensional version has a long history as an effective theory of QCD at intermediate energies.

Having highlighted the model's zero temperature properties, let us now discuss what we know of its thermodynamics. To leading order in $1 / N_{f}$ the effective potential has the same form as the discrete symmetry case with the replacement $\sigma^{2} \rightarrow \sigma^{2}+\vec{\pi}^{2}$. This implies that chiral symmetry remains broken up to a critical temperature $T_{c}=\frac{M}{2 \ln 2}(M$ is the zero temperature fermion mass) and that symmetry restoration at this temperature is associated with the fermions becoming massless. This conclusion is expected to be valid only when $N_{f}$ is strictly infinite, i.e. when the fluctuations of the bosonic fields are neglected, since otherwise it runs foul of Coleman-Mermin-Wagner (CMW) theorem [4] which states that in $2+1$ dimensional systems a continuous symmetry must be manifest for all $T>0$. This behaviour may be understood by noting that, in the language of spin models, a domain wall separating regions of oppositely oriented magnetisation has an energy which does not increase with the size of the system. If a wall has length $l$ and thickness $t$, the energy is $\sim l / t$, since the magnetisation is allowed to interpolate continuously [4]. This remains finite even as $l, t \rightarrow \infty$. The physics is comparable, therefore, to that of the Ising chain, where a kink also costs only finite energy. At $T=0^{+}$the magnetisation changes discontinuously, but there is no latent heat as the domains can grow to be very large. A simple estimate of the entropy [4] reveals that the domain size grows exponentially with the inverse temperature. Studies of the critical properties of the $2+1 d$ NJL models with $Z_{2}$ [5] and $U(1)$ [1, 2, 3] chiral symmetries at nonzero temperature have shown that the thermally induced phase transition of the $Z_{2}$-symmetric model belongs to the universality class of the two-dimensional Ising model and that the $U(1)$-symmetric model has the same phase structure as the two-dimensional $X Y$ model. These results are in accordance with the dimensional reduction scenario, 
which predicts that the long range behaviour at the chiral phase transition is that of the $(d-1)$ spin model with the same symmetry. This is because the IR region of the system is dominated by the zero Matsubara mode of the bosonic field.

In the $S U(2) \otimes S U(2)$-symmetric model, one expects to observe fermion mass generation in the absence of symmetry breaking at finite temperature, as in the case of the

$U(1)$ model. The local amplitude $\rho=\sqrt{\sigma^{2}+\vec{\pi} \cdot \vec{\pi}}$ is neutral under $O(4)$ rotations and may therefore be nonzero without breaking the symmetry. Hence, it is possible to have a dynamically generated fermion mass $M(T) \sim \rho$ whose value may be comparable to the naive prediction of the large- $N_{f}$ approach. This was demonstrated in $d=(1+1)$ in [6], in which results from an exactly soluble fermionic model were generalised to the $U(1)$ symmetric Gross-Neveu model. As was argued in [3], at $T \neq 0$ the fermionic spectral function is modified to a branch cut, implying that the propagating fermion constantly emits and absorbs massless scalars and hence has an indefinite chirality. The physical fermion may then be a superposition of positive and negative chirality and therefore be neutral under chiral transformations. It is in this way that one anticipates mass generation despite manifest symmetry.

In this study we have carried out lattice simulations with $N_{f}=4$. After briefly discussing the lattice formulation of the model, we present results of simulations at $T=0$ from which we have extracted the bulk critical exponents $\beta_{\text {mag }}$ and $\nu$. For $T \neq 0$ we have attempted to study the issue of mass generation by measuring the so-called screening or "spatial" mass $M_{s}(T)$ and the pole or "temporal" mass $M_{t}(T)$. In order to better understand the relationship between the two we carried out a study of the lattice dispersion relation. Finally, simulation on lattices with various temporal extents helps to illustrate the nature of finite size and lattice discretisation effects. Measuring the pressure and making comparison with the Stefan-Boltzmann limit was found to elucidate the matter further.

\section{Lattice Formulation}

In its bosonised form, the model may be formulated on the lattice using the action

$$
S=\sum_{i=1}^{N} \sum_{x}\left[\bar{\chi}_{i} M[\sigma, \vec{\pi}] \chi_{i}+\bar{\zeta}_{i} M^{*}[\sigma, \vec{\pi}] \zeta_{i}\right]+\frac{2 N}{g^{2}} \sum_{\tilde{x}}\left(\sigma^{2}+\vec{\pi} \cdot \vec{\pi}\right)
$$

where $\chi, \bar{\chi}, \zeta$ and $\bar{\zeta}$ each represent $N$ independent Grassmann-valued staggered fermionic fields defined on lattice sites $x$, and the auxiliary bosonic fields $\sigma$ and $\vec{\pi}$ are defined on dual lattice sites $\tilde{x}$. The fermion kinetic operator is given by

$$
\begin{aligned}
M_{x y}^{p q}[\sigma, \vec{\pi}] & =\frac{1}{2} \delta^{p q}\left[\left(\delta_{y x+\hat{0}}-\delta_{y x-\hat{0}}\right)+\sum_{\nu=1,2} \eta_{\nu}(x)\left(\delta_{y x+\hat{\nu}}-\delta_{y x-\hat{\nu}}\right)\right] \\
& +\frac{1}{8} \delta_{x y} \sum_{\langle\tilde{x}, x\rangle}\left(\sigma(\tilde{x}) \delta^{p q}+i \epsilon(x) \vec{\pi}(\tilde{x}) \cdot \vec{\tau}^{p q}\right)
\end{aligned}
$$


where $\langle\tilde{x}, x\rangle$ represents the set of 8 dual lattice sites neighbouring $x$, and the symbols $\eta_{\nu}(x)$ and $\epsilon(x)$ are the phases $(-1)^{x_{0}+\ldots+x_{\nu-1}}$ and $(-1)^{x_{0}+x_{1}+x_{2}}$ respectively. $p, q$ are the internal $S U(2)$ isospin indices, which we include explicitly. Making the replacement $\gamma_{5} \rightarrow \epsilon(x)$, the symmetries defined in the previous section are still observed, i.e. the $S U(2) \otimes S U(2)$ chiral symmetry remains exact [7], which is not the case in lattice QCD. With this replacement, the projection operators $P_{L, R} \rightarrow P_{e, o}$ now project onto even and odd sub-lattices respectively.

This choice of action corresponds to using a functional weight of $\operatorname{det} M^{\dagger} M$. This doubles the fermionic degrees of freedom, but does allow one to perform simulations using a hybrid Monte Carlo algorithm, which has the advantage of being exact. Further doubling due to the use of staggered fermions in three dimensions leads to a total of $N_{f}=4 N$ continuum fermion flavors. Trajectory lengths were sampled from a Poisson distribution with a mean of approximately 1.0. This ensures the ergodicity of the ensemble and reduces the autocorrelation of successive configurations.

Simulations were carried out on lattices with $L_{s}^{2} \times L_{t}$ sites, separated by lattice spacing $a \sim 1 / \Lambda$, where $\Lambda$ is the ultra-violet cut-off. In Euclidean spacetime, temperature is identified with the inverse temporal extent, i.e. $T=1 /\left(L_{t} a\right)$. The temperature of the system may be altered, therefore, by varying either $\beta \equiv 1 / g^{2}$, which amounts to varying the lattice spacing, or by varying $L_{t}$. The effective zero temperature limit is reached if one chooses $L_{t} \simeq L_{s} \gg 1$ and cut-off dependent effects may be explored by varying $\beta$. To reach the continuum limit one has to satisfy the following two conditions: $\Lambda \gg T$ and $\Lambda \gg m$, where $m$ is a mass scale inversely associated with the correlation length $\xi$ of the fluctuations of the order parameter. The condition $\Lambda \gg T$ requires a lattice with sufficiently large $L_{t} \gg 1$. This condition not being met results in the sum over the Matsubara frequencies being truncated at too small a value. The second condition, $\Lambda \gg m$, implies that the coupling $\beta$ should be tuned toward its critical value at which the correlation length $\xi \gg 1$. Furthermore, the condition $L_{s} \gg \xi$ should be satisfied in order to minimise finite size effects and obtain results which approximate the thermodynamic limit.

\section{Results}

\subsection{Zero Temperature}

In order to study the chiral symmetry of the model first at $T=0$ and then at $T>0$ we work in the chiral limit, i.e. we choose not to introduce a bare fermion mass $m_{0}$ into eq. (3). Without the benefit of this interaction, the direction of symmetry breaking changes over the course of the simulation such that $\Sigma \equiv \frac{1}{V} \sum_{\tilde{x}} \sigma(\tilde{x})$ and $\vec{\Pi} \equiv \frac{1}{V} \sum_{\tilde{x}} \vec{\pi}(\tilde{x})$ (where $V$ is the lattice volume) average to zero over the ensemble. It is in this way that the absence of spontaneous chiral symmetry breaking on the finite lattice is enforced. 
Instead, we define an effective order parameter

$$
\Phi \equiv\left\langle\sqrt{\Sigma^{2}+\vec{\Pi} \cdot \vec{\Pi}}\right\rangle
$$

which is a projection onto the direction of symmetry breaking for each configuration. Although this definition allows one to distinguish between the chirally broken and unbroken phases, it should be noted that the two definitions of the order parameter, $\Phi$ and $\left.\langle\sigma\rangle\right|_{m_{0} \rightarrow 0}$, differ by a factor of $1 / \sqrt{V}$ as it is impossible to disentangle the chiral order parameter field in $\Phi$ from the Goldstone modes. We also measure the fermion correlation function for temporal separation $t$;

$$
\begin{array}{cl}
C(t)=A_{t}\left[e^{-M t}+e^{-M\left(L_{t}-t\right)}\right] & \text { if } t=\text { odd } \\
C(t)=0 & \text { if } t=\text { even }
\end{array}
$$

which allows one to extract the fermion pole mass $M$. The fact that $C(t=$ even $)$ averages to zero over the ensemble reflects the fact that chiral symmetry is not truly broken on our finite size system, as manifest chiral symmetry implies that the $G_{o o}$ and $G_{e e}$ components of the staggered fermion propagator should vanish.

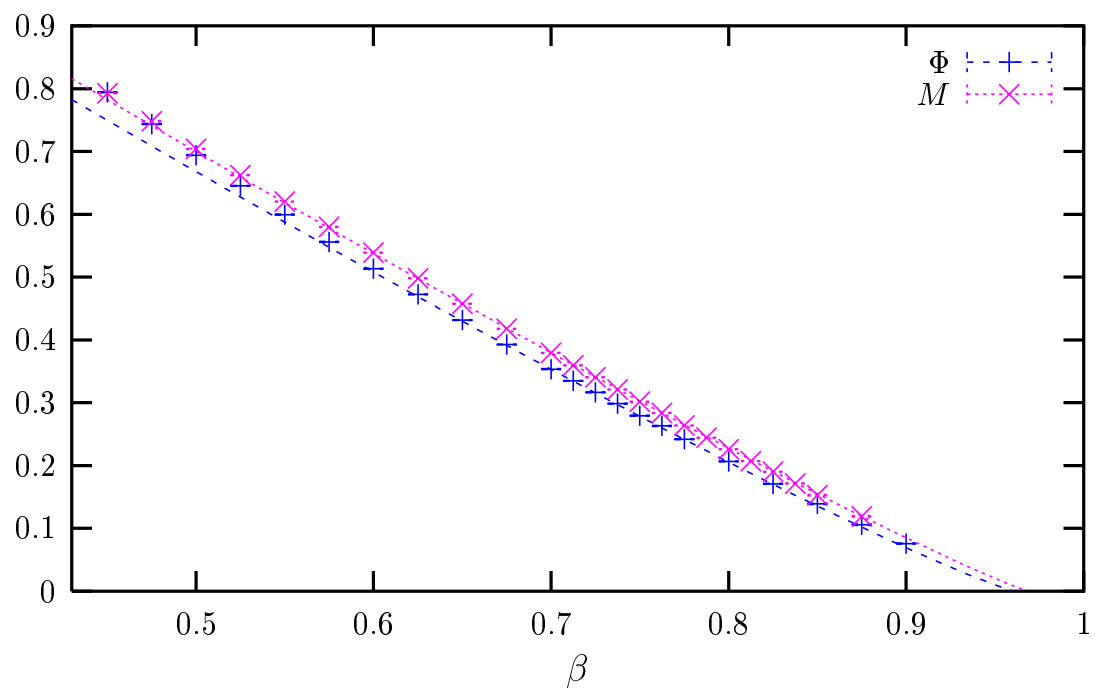

Figure 1: Effective order parameter and fermion mass as functions of $\beta$ on $36^{3}$ and $48^{3}$ lattices.

We simulated the model on a $36^{3}$ lattice with repeated simulations on a $48^{3}$ lattice near the critical coupling $\beta_{c}^{\text {bulk }}$ in order to detect and control finite size effects. Approximately 1000 equilibrated trajectories were generated for each point. Fitting these results to the scaling forms $\Phi=a_{1}\left(\beta_{c}^{\text {bulk }}-\beta\right)^{\beta_{\text {mag }}}$ and $M=a_{2}\left(\beta_{c}^{\text {bulk }}-\beta\right)^{\nu}$ we were able to extract the critical exponents $\beta_{\text {mag }}$ and $\nu$. As $\beta \rightarrow 0$, the space-time lattice becomes large and coarse and the $\mathcal{O}(a)$ lattice discretisation effects become significant. As $\beta \rightarrow \beta_{c}^{\text {bulk }}$ the decreasing lattice spacing causes the lattice to become less coarse, but finite volume 
effects become dominant. True scaling behaviour is limited, therefore, to a particular window, which we choose as $0.650 \leq \beta \leq 0.825$ by demanding that results of the fits be stable with respect to the adding and subtracting of individual points. The measured values of the exponents were $\beta_{\text {mag }}=1.12(2)$ and $\nu=1.10(2)$ with $\beta_{c}^{\text {bulk }}=0.961(5)$ and $0.969(3)$ respectively. Data for $\Phi$ and $M$ versus $\beta$ are plotted with the fitted scaling functions in Fig. 11.

Our results for $\nu$ and $\beta_{\text {mag }}$ are consistent with the $1 / N_{f}$ expansion results calculated in 8$]$;

$$
\begin{aligned}
\beta_{\mathrm{mag}} & =1+\frac{3}{2}\left(\frac{4}{\pi^{2}}\right) \frac{1}{N_{f}}+\mathcal{O} \frac{1}{N_{f}^{2}} \sim 1.15 \\
\nu & =1+\frac{4}{3}\left(\frac{4}{\pi^{2}}\right) \frac{1}{N_{f}}+\mathcal{O} \frac{1}{N_{f}^{2}} \sim 1.14
\end{aligned}
$$

where in our simulations $N_{f}=4$. The fact that we observed next-to-leading order corrections, which were not observed in studies of the $Z_{2}$ [8] and $U(1)$ [3] models, is because the $\mathcal{O}\left(1 / N_{f}\right)$ corrections in eq. (7) and eq. (8) are slightly larger than in the models of lower symmetry. The fact that there are more light mesons that mediate the interaction in the $S U(2) \otimes S U(2)$-symmetric model than in the $Z_{2}$ and $U(1)$ models may be the source for the larger deviation from the mean field values, which are $\beta_{\text {mag }}=\nu=1$ for all three symmetries. More accurate values of the exponent could be extracted by performing detailed finite size scaling analyses in the vicinity of the critical point.

In addition to the extraction of critical exponents, the studies presented here provide a length scale, namely the fermion mass $M$, with which one can present nonzero temperature results in a cut-off independent fashion. This is performed in the next section.

\subsection{Nonzero Temperature}

In order to study the model at nonzero temperature we performed simulations on lattices with a constant temporal extent $L_{t}=4$ and varying spatial volumes $V_{s}=$ $30^{2}, 50^{2}, 100^{2}, 150^{2}$ and $220^{2}$. The temperature was varied continuously by varying $\beta<\beta_{c}^{\text {bulk }} \sim 0.965$, at which point $a \rightarrow 0$ and $T \rightarrow \infty$.

The effective order parameter is plotted against temperature in Fig. 2. The temperature is normalised by a factor of the zero temperature pole mass $M(0)$, in order to make results cut-off independent as previously discussed. At high temperature, $\Phi$ is consistent with zero whilst at low temperature there are significant finite size effects. These effects are a signal of the correlation length diverging as one approaches the transition at $T=0^{+}$and the condition $L_{s} \gg \xi$ not being met. Once $\xi \sim L_{s}$ one is unable to observe a manifest symmetry. Our results are consistent with the expectation of chiral symmetry restoration in the infinite volume limit for $T \neq 0$.

This picture is supported by the results shown in Fig. 3, in which the susceptibility of the order parameter

$$
\chi=V\left(\left\langle|\Phi|^{2}\right\rangle-\langle|\Phi|\rangle^{2}\right)
$$




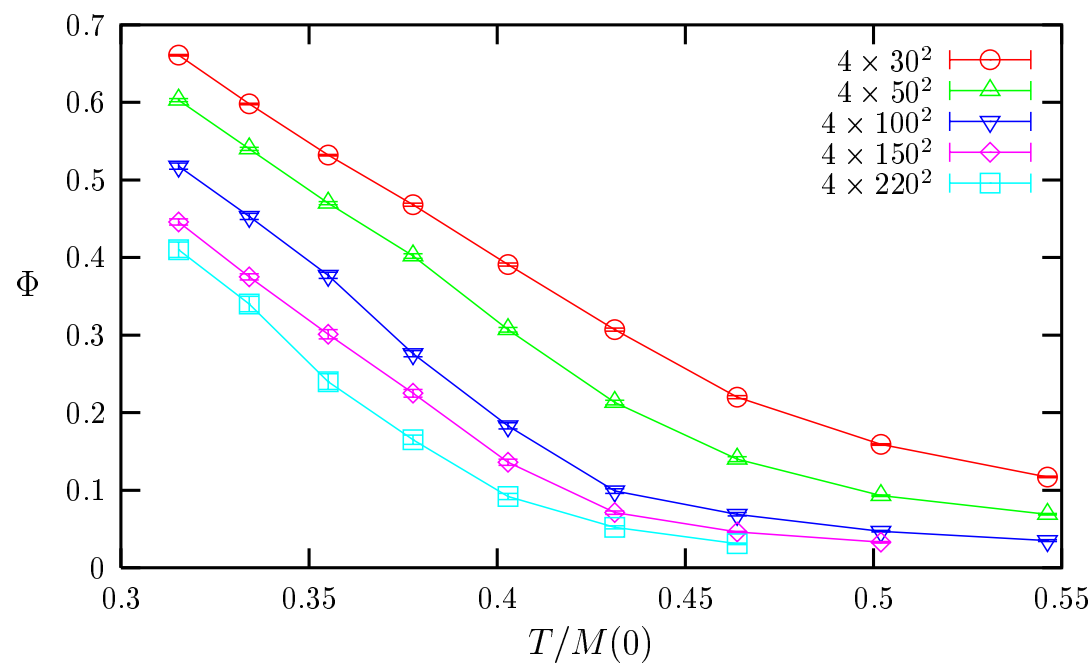

Figure 2: Effective order parameter vs. normalised temperature for various spatial volumes.

is plotted as a function of temperature. As spatial volume is increased, the pseudocritical temperature $T_{c}\left(L_{s}\right)$, i.e. the position of the peak in susceptibility, decreases, supporting a transition at $T=0^{+}$in the infinite volume limit. Finite size effects are again very large in the low $T$ regime, which is again consistent with the expectation that the correlation length diverges as $T \rightarrow 0$. It is elucidating to compare our results for $\Phi$ and $\chi$ with those from simulations of the $U(1)$-symmetric model [3], where it was shown that a Berezinskii-Kosterlitz-Thouless transition separates two chirally symmetric phases, one critical and one with finite correlation length. The main observations are: (a) in the $U(1)$ model the finite size effects in the low $T$ phase are larger than in the $S U(2) \otimes S U(2)$ model because the correlation length is infinite for any $T \leq T_{B K T}$; (b) the positions of the susceptibility peaks in the $U(1)$ model converge to a nonzero $T=T_{B K T}$.

In order to address the issue of mass generation with $L_{t}=4$, we cannot directly extract the fermion mass from temporal correlators via eq. (6) as there are no longer enough temporal separations over which to carry out a fit. Instead, by studying the decay of spatial correlators, we extract the so-called screening or "spatial" mass $M_{s}(T)$ via

$$
\begin{array}{cl}
C(s)=A_{s}\left[e^{-M_{s} s}+e^{-M_{s}\left(L_{s}-s\right)}\right] & \text { if } s=\text { odd } \\
C(s)=0 & \text { if } s=\text { even. }
\end{array}
$$

For free, massless fermions we expect this quantity to be the lowest fermion Matsubara mode, given in the continuum by $\omega_{0}^{\text {cont }}=\pi T$, and on the lattice by $\omega_{0}^{\text {lat }}=$ $\sinh ^{-1}\left[\sin \left(\pi / L_{t}\right)\right] ; \omega_{0}^{\text {lat }} \approx 0.658$ for $L_{t}=4$. In the low temperature phase of the large- $N_{f}$ limit the "temporal" or pole fermion mass $M_{t}(T)$ is related to the screening or "spatial" mass $M_{s}(T)$ via $M_{s}^{2}=\omega_{o}^{2}+M_{t}^{2}$, as the effects of the interaction are absorbed by the dynamically generated mass. If $1 / N_{f}$ effects are taken into account, however, the relation between $M_{t}$ and $M_{s}$ can be less trivial. In particular, at nonzero temperature the 


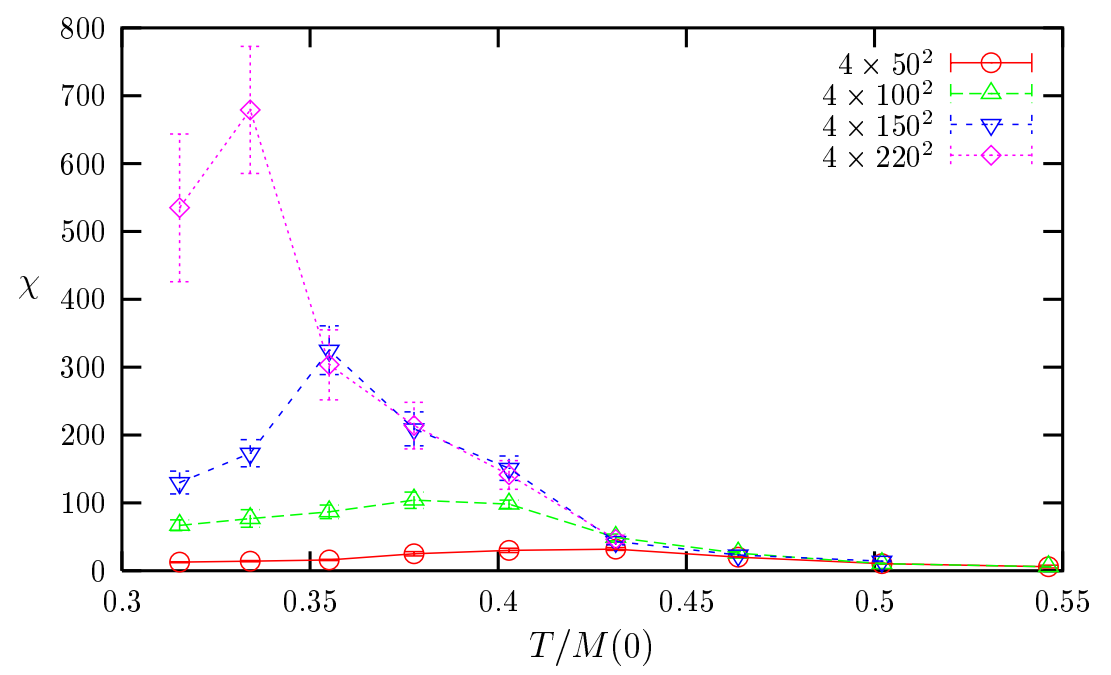

Figure 3: Susceptibility vs. normalised temperature for various spatial volumes.

fermionic dispersion relation is expected to reflect the breaking of Lorentz invariance via a temperature dependent vacuum polarisation term $\Pi(\vec{k}, T)$ :

$$
E^{2}(\vec{k}, T)=M^{2}(0)+\vec{k}^{2}+\Pi(\vec{k}, T) .
$$

Temperature effects can be absorbed into the thermal mass $M(T)$ and a coefficient $A(T)$ which can differ from one, implying a temperature dependent modification to the speed of light. The latter effect is expected to be significant for $|\vec{k}| \ll T$, allowing one to write $\Pi(\vec{k}, T)=\alpha^{2}(T) \vec{k}^{2}+\mathcal{O}\left(\vec{k}^{4}\right)$. The dispersion relation becomes

$$
E^{2}(\vec{k}, T)=M^{2}(T)+A^{2}(T) \vec{k}^{2}
$$

where $A^{2}=\alpha^{2}+1$. It is simple to show that the relationship between the screening and the pole mass is given by $A^{2} M_{s}^{2}=M_{t}^{2}+\omega_{0}^{2}$, implying that a knowledge of $M_{s}$ alone is not sufficient to reach a conclusion about the magnitude of the dynamical fermion mass $M_{t}$. This issue is investigated later in this section.

Results from the measurement of the screening mass from the $L_{t}=4$ simulations on various spatial volumes are presented in Fig. 4. The first observation to be made is that at low temperature $M_{s}(T)$ is significantly larger than $\omega_{0}^{\text {lat }}$. It is an interesting aside to note that, as was the case with the $U(1)$ model [3], the magnitude of the screening mass in our simulations is comparable to the values extracted from simulations of the $Z_{2}$-symmetric model [5] where mass generation is ascribed to orthodox chiral symmetry breaking. Fig. 1 shows the mass to be size independent, even though, as we have seen from Fig. 2, the order parameter $\Phi$ has large finite size effects for all $T>0$. It is also clear from Fig. 1 that $M_{s}$ does not approach a value equal to $\omega_{0}^{\text {lat }}$ in the limit that $T \rightarrow \infty$. We attempted to understand this by measuring the fermion mass on lattices with different temporal extents $L_{t}=8,16$ and 24 and for a range of values of the coupling $\beta$. The 


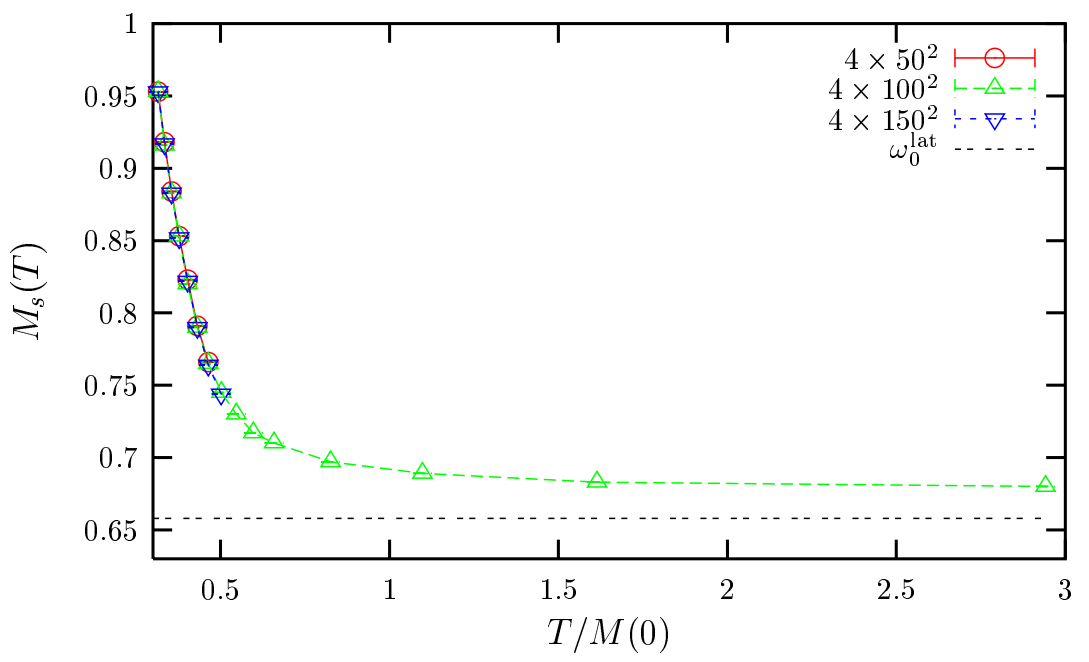

Figure 4: Screening mass vs. normalised temperature. The horizontal line represents the lowest Matsubara mode.

screening mass measured on lattices with three different values of $L_{t}$ are presented in Fig. 5. The data are normalised by a factor of $\omega_{0}^{\text {lat }}$ to make it possible to plot the results on the same axes. At low temperature there seems to be no $L_{t}$ dependence whilst at high temperature we see that the discrepancy between $M_{s}(T)$ and $\omega_{0}^{\text {lat }}$ is reduced as $L_{t}$ is increased. The effect of finite $L_{t}$ is that only a finite number of Matsubara modes are present on the lattice, and when the fermion screening mass becomes comparable with the lowest Matsubara mode $M_{s}(T) \sim \omega_{0}^{\text {lat }}$, discretisation artifacts become significant. It is clear that this effect is only apparent when comparison is made with large- $L_{t}$ data.

In order to gain insight into the effects of finite $L_{t}$ and finite physical volume (which at nonzero temperature is translated into finite asymmetry ratio $L_{s} / L_{t}$ ) we studied the pole mass on different lattice sizes $8 \times 48^{2}, 16 \times 48^{2}, 16 \times 96^{2}$ and $24 \times 72^{2}$. The results of the pole mass normalised with respect to $M(0)$ are presented in Fig. 6. At this point we remind the reader that in order to increase $T$, we decrease the lattice spacing $a$, which is achieved by tuning the coupling $\beta$ toward the bulk critical point $\beta_{c}^{\text {bulk }}$. Comparing curves with the same asymmetry ratios and different $L_{t}$, such as those extracted from data on lattice sizes $8 \times 48^{2}$ with $16 \times 96^{2}$ and $16 \times 48^{2}$ with $24 \times 72^{2}$, we see that the finite $L_{t}$ effects become large as $T$ increases. As in Fig. 5, this is due to the fermion screening mass becomes comparable with the lowest Matsubara mode. Similarly, the effects of finite spatial extent become more pronounced with temperature, since finite size effects become large when the fermion correlation length is comparable with the spatial extent of the lattice $\xi \sim M_{t}^{-1} \sim L_{s}$. We have plotted the same data with a different normalisation in Fig. 7 and fitted to the form $M_{t}(T) / T=\alpha_{1}(T / M(0))^{\alpha_{2}}$. We chose to fit only to data with $T / M(0) \lesssim 0.5$, as we know from Fig. 6 that in this region finite size and discretisation errors are not severe. This choice is justified by noting that in Fig. 0 the data at large $T$ deviate from the fitting line. We found a weak temperature dependence, with $0.68<\alpha_{1}<0.85$ and $-1.25<\alpha_{2}<-1.12$ over the four fits. This is 


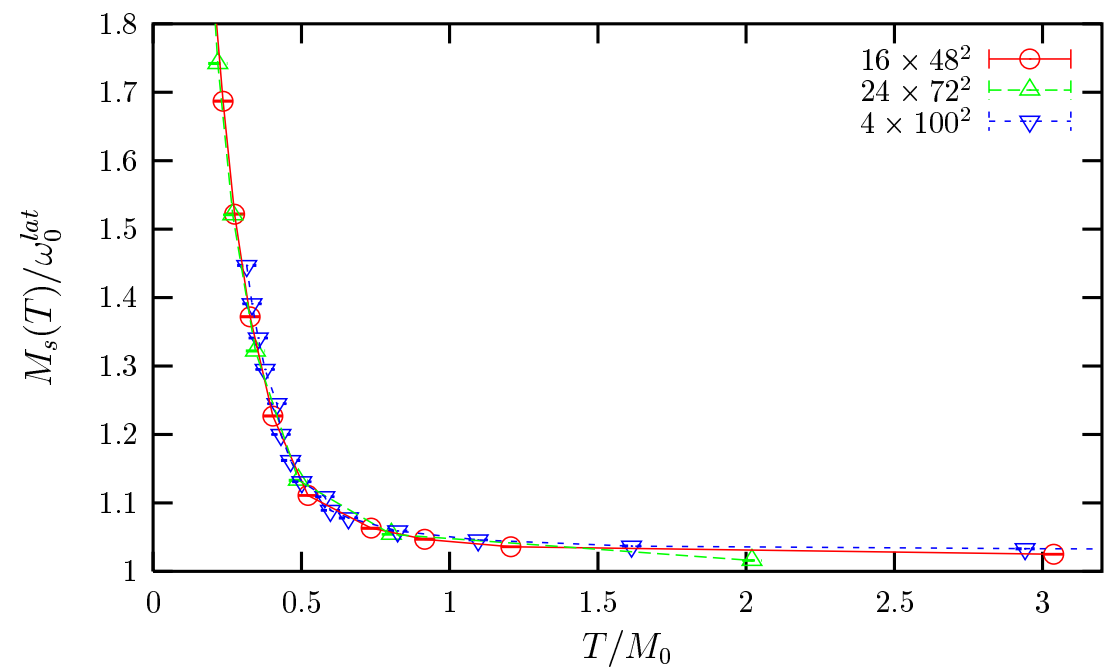

Figure 5: Screening mass vs. normalised temperature. In order to compare different $L_{t}$ we normalise by a factor of $\omega_{0}^{\text {lat }}$.

consistent with $M_{t} \rightarrow 0$ as $T \rightarrow \infty$. In the future, it would be interesting to compare this result with next-to-leading order $1 / N_{f}$ calculations.

As previously discussed, in order to better understand the temperature dependence of eq. (12) we have studied the fermion correlation function at nonzero momentum $k=2 \pi n / L_{s}\left(n=0,1,2, \ldots, L_{s} / 4\right)$ in the spatial direction and used the energy $E(k)$ extracted from eq. (6) to map out the fermion dispersion relation. Due to the periodicity of the lattice in spatial dimensions and the doubling of fermion species, these dispersion relations are symmetric around $k=\pi / 2$. We fitted data from the zero and nonzero temperature phases to the lattice free fermion dispersion relation [9]

$$
\sinh ^{2} E=A^{2} \sin ^{2} k+\sinh ^{2} M_{t} .
$$

Results from the hot phase on a $16 \times 48^{2}$ lattice are shown with the fitted forms in Fig. 8. The masses extracted from fits to $E(k)$ at both $T=0$ and $T>0$ are consistent with those extracted from the zero momentum correlators and yield values of $A \simeq 1$ to within less than 3\%. This implies that the principal physical effect of the hot medium is to generate a nonzero thermal mass, rather than to renormalise the speed of light. Furthermore, our results show that any extra factors not included in the free-field ansatz eq. (13) are negligible. Similar results were extracted by fitting the dispersion relation to data from $16 \times 96^{2}$ and $24 \times 72^{2}$ lattices. The $A \simeq 1$ result provided additional evidence that the nonzero screening mass extracted from our simulations with $L_{t}=4$ implies that the fermion pole mass is nonzero. We see no modifications to the low momentum part of the dispersion relation, expected to have two branches at $T>0$ [10]. These branches, corresponding to two types of quasi-particle excitations (the fermion and the plasmino) are not visible in our simulations.

Finally, to gain further insight into the thermodynamics of the model, we studied 


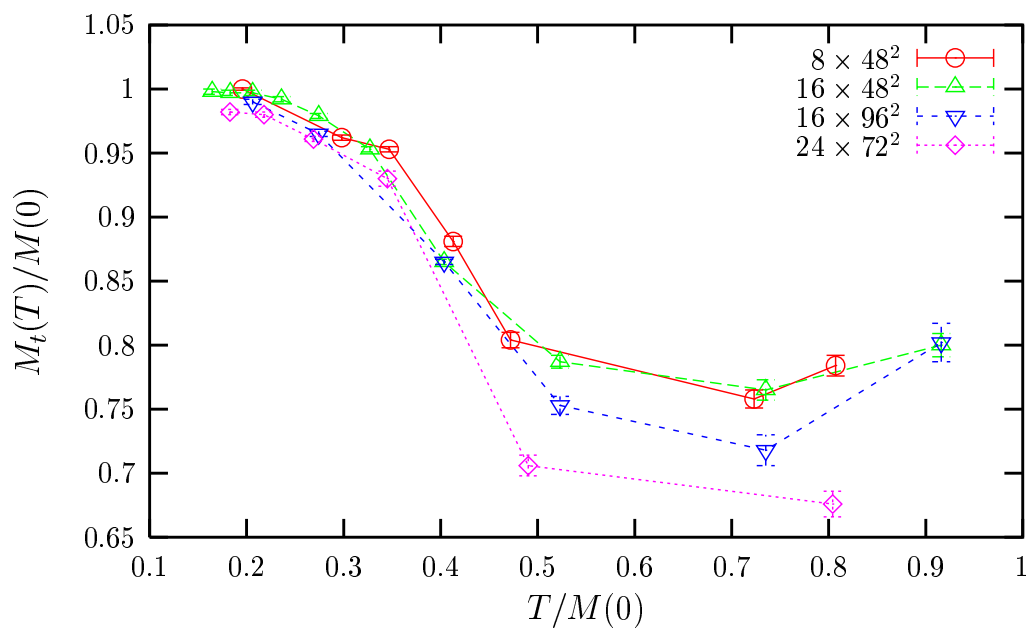

Figure 6: $M_{t}(T) / M(0)$ vs. $T / M(0)$.

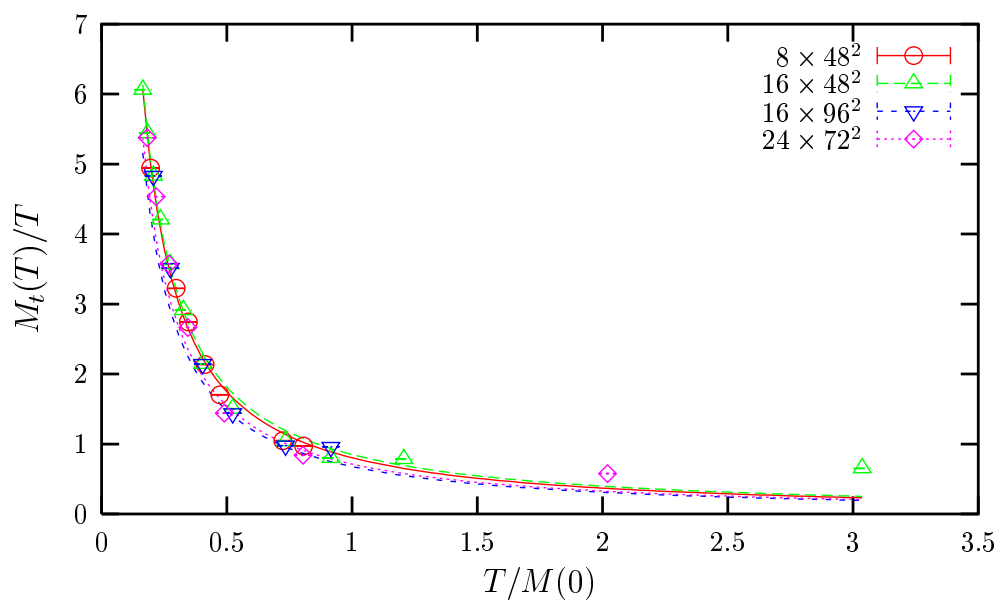

Figure 7: $M_{t}(T) / T$ vs. $T / M(0)$.

the equation of state by measuring the pressure as a function of temperature using the integral method [1]. For homogeneous systems the pressure $P$ is given by

$$
\left.P \equiv \frac{\partial \ln \mathcal{Z}}{\partial V}\right|_{T}=\frac{\ln \mathcal{Z}}{V}=-f,
$$

where $f$ is the free energy density. On the lattice the logarithm of the partition function is calculated from the expectation value of the bosonic part of the action $S_{\text {bos }} \equiv 2 N \sum_{\tilde{x}}\left[\sigma^{2}(\tilde{x})+\vec{\pi}(\tilde{x}) \cdot \vec{\pi}(\tilde{x})\right]$. Since the derivative with respect to the bare coupling $\beta$ is

$$
-\frac{\partial Z}{\partial \beta}=\langle S\rangle,
$$




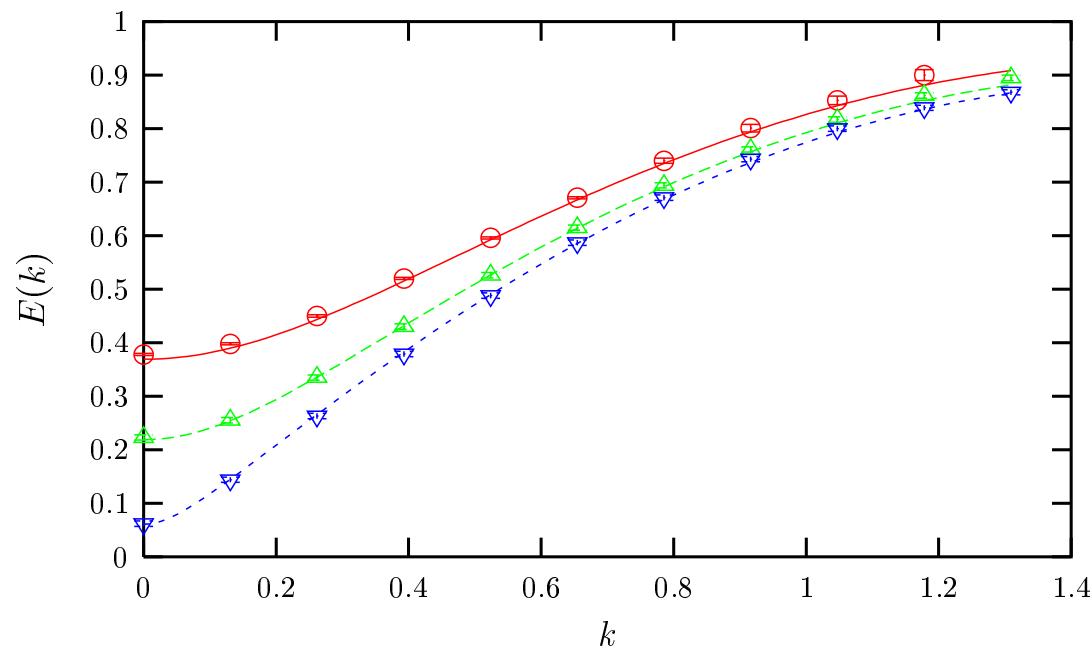

Figure 8: Fermionic dispersion relation vs. momentum $k$. The rescaled temperature $T / M(0)$ decreases from 0.165 (top) to 0.735 (bottom).

the physical free energy density can obtained from

$$
f=\frac{1}{V} \int_{\beta_{0}}^{\beta} \mathrm{d} \beta^{\prime}\left[\left\langle S_{b o s}\right\rangle_{0}-\left\langle S_{b o s}\right\rangle_{T}\right]
$$

The free energy density is normalised by subtracting the vacuum contribution calculated at $T=0$. The pressure was calculated from data on the $4 \times 100^{2}$ lattice whilst the vacuum expectation value of $S_{\text {bos }}$ was calculated from data on $36^{3}$ and $48^{3}$ lattices. A lattice large- $N_{f}$ calculation of $P$, including the calculation of the lattice Stefan-Boltzmann limit, is outlined in Appendix A. This result calculated on a $4 \times 100^{2}$ lattice is compared with the result extracted from simulations with $N_{f}=4$ on the same lattice size in Fig. 9 . The statistical error in the pressure extracted from simulation data is approximately $5 \%$ of the value of $P$ for all $T$. It is striking that the value of $P / T^{3}$ extracted from the simulations doesn't reach the Stefan-Boltzmann limit as $T \rightarrow \infty$, an effect found to be independent of the spatial volume. We believe that this is simply another manifestation of the effect that causes the screening mass not to approach $\omega_{0}^{\text {lat }}$ as $T \rightarrow \infty$. We interpret it as a renormalisation of discretisation artifacts by $1 / N_{f}$ corrections. Unfortunately, our data on lattices with $L_{t}>4$ were not sufficient to study the equation of state because the fact that as $L_{t}$ becomes large the two contributions to eq. (16) converge, in conjunction with the normalisation factor of $T^{-3}=L_{t}^{3}$ causes statistical errors to swamp any signal in the data.

Drawing the information in this section together, our results provide clear evidence for the fermions acquiring nonzero mass in the absence of chiral symmetry breaking. In order to be certain whether mass generation switches off at some value of $T=T^{*}$ or whether it goes to zero asymptotically at $T \rightarrow \infty$, we would need to simulate the model on lattices with much larger temporal and spatial extents. 


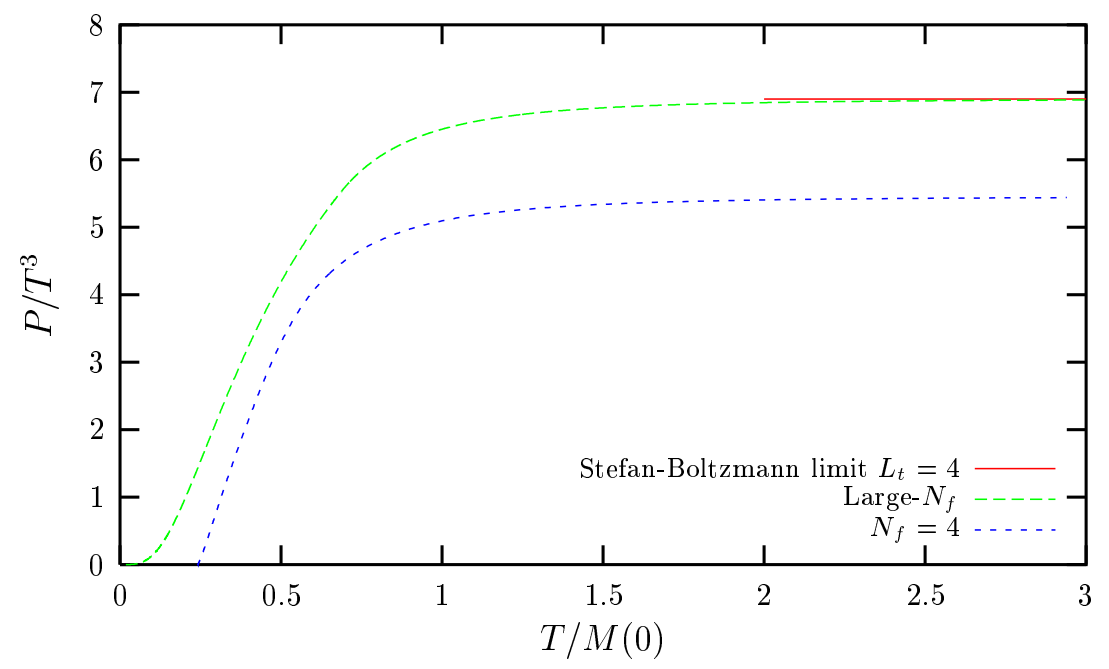

Figure 9: The pressure calculated from the lattice gap equation and from numerical simulations. In both cases the lattice size is $4 \times 100^{2}$.

\section{Conclusions}

In this paper we have presented a thermodynamic lattice study of the $2+1$ dimensional $S U(2) \otimes S U(2)$-symmetric NJL model. We find the model to have manifest chiral symmetry for all $T \neq 0$ in accordance with the CMW theorem. As temperature is reduced, the susceptibility of the order parameter begins to diverge and is consistent with there being a phase transition at $T=0^{+}$. This should be contrasted with the predictions of the large- $N_{f}$ limit, in which fluctuations of the bosonic fields are neglected and chiral symmetry remains broken up to a critical temperature $T_{c}=\frac{M(0)}{2 \ln 2}$. In this limit, the breaking of chiral symmetry is associated with the generation of fermion mass in the standard way. We have demonstrated non-perturbatively, that in agreement with large- $N_{f}$ there is mass generation at $T \neq 0$ in this model.

By studying the so-called "screening" mass $M_{s}(T)$, we have shown that at low but nonzero temperature $M_{s} \gg \omega_{0}$. With a temporal extent of $L_{t}=4$, we observed that the spatial mass fails to approach $\omega_{0}$ in the $T \rightarrow \infty$ limit. This effect is also observed in the equation of state, where as $T \rightarrow \infty$, the pressure fails to approach the Stefan-Boltzmann limit. This is due to the fact that when $L_{t}$ is small there are only a small number of Matsubara modes available, an effect which becomes noticeable at high temperatures, where $M_{s} \sim \omega_{0}$. Results from measurements of $M_{s}$ on lattices with $L_{t}>4$ have shown this to be a discretisation effect which is reduced when $L_{t} \gg 1$. By studying the fermion dispersion relation, we have also shown that any temperature dependent modification to the relationship between $M_{s}$ and $M_{t}$ is negligible, implying that we have unambiguous evidence that at $T>0$ we observe mass generation despite the lack of chiral symmetry breaking. It is interesting to note that the value of $M_{s}(T)$ is of the same magnitude as it is in the model with $Z_{2}$ chiral symmetry [3], in which mass generation is ascribed to conventional symmetry breaking. To support our evidence of mass generation from 
the measurement of $M_{s}$ we have also measured the pole mass directly on lattices with $L_{t} \gg 1$. At high temperatures we observe large finite size effects as the correlation length of the low-mass fermion becomes comparable to the spatial extent of the system. At low temperatures, however, we observe that the mass moves smoothly toward its zero temperature value. As with the results from the $U(1)$ model, we take this as further support for Witten's statement [6] that when interpreted correctly, the large$N_{f}$ expansion is a reliable guide to the properties of the model. Although the CMW theorem forbids the large- $N_{f}$ prediction of symmetry breaking at $T>0$, the prediction of a dynamically generated fermion mass is adhered to. In this case the propagating fermion constantly emits and absorbs massless scalars and hence has indefinite chirality. As was argued in [3], the $T \neq 0$ fermionic spectral function is modified to a branch cut. In this model we observe mass generation without phase coherence, i.e. we have a pseudogap phase for $T>0$. At this stage, however, we cannot say whether there is a phase transition at some temperature $T^{*}$ or the fermion mass goes asymptotically to zero as $T \rightarrow \infty$. In order to study this regime (corresponding to $a \rightarrow 0$ ) it is necessary to perform new simulations on lattices with larger $L_{t}$ and $L_{s}$.

To put these results into context, we note that the observation of mass generation without chiral symmetry breaking in $2+1 d$ adds to the already rich and interesting phase diagram of the $S U(2) \otimes S U(2)$ NJL model [12]. At high baryon chemical potential and zero temperature a complementary phenomenon is known to occur. Monte Carlo simulations have shown there to be a "thin film" superfluid phase with long range phase coherence, but no mass gap is generated about the Fermi surface [13]. Although similar in nature to the $2 d X Y$ spin model, a unique critical exponent seems to put the high- $\mu$ phase in a new universality class. In $3+1$ dimensions, the high- $T$ low- $\mu$ phase shows conventional symmetry restoration via a $T \neq 0$ transition and the high- $\mu$ low- $T$ phase appears to be that of a traditional BCS superfluid. A study of the region at high- $\mu$ and above the critical temperature, however, shows it to behave as if in a pseudogap phase [14]. Analogous to strong coupling superconductors there are two distinct critical temperatures: (a) $T^{*}$ below which there is manifest baryon number symmetry but no mass gap $\Delta$, and (b) $T_{c}$ below which normal superfluidity occurs. This is particularly interesting, as it provides evidence for mass gap generation without phase coherence in a phenomenologically relevant number of dimensions.

\section{Acknowledgements}

Costas G. Strouthos was supported by a Leverhulme Trust grant. Discussions with Simon Hands are greatly appreciated.

\section{Appendix A: Large $-N_{f}$ calculation of the Pressure}

In this appendix we outline a derivation for the pressure in the large- $N_{f}$ limit on the lattice. In section 2 we define our model in terms of staggered fermion fields which are 
defined on sites $x$ of the space-time lattice. In $d$ dimensions, fermion doubling gives rise to $2^{d}$ degrees of freedom per naive lattice flavor, which in the staggered formalism are interpreted as $2^{d} / 4$ "tastes"' of Dirac spinor. This is easier to understand if we redefine the fields on a blocked lattice, with each site $y$ associated with $2^{d}$ sites of the original lattice [15]. The kinetic operator then tends to the continuum Dirac operator in the limit $a \rightarrow 0$, with a pole in the propagator only at zero momentum. We apply this formalism, which is outlined for an odd number of dimensions in [16], in the case that $d=3$. Defining the $2 \times 2$ matrices $\Gamma_{A}=\tau_{1}^{A_{1}} \tau_{2}^{A_{2}} \tau_{3}^{A_{3}}$ and $B_{A}=\left(-\tau_{1}\right)^{A_{1}}\left(-\tau_{2}\right)^{A_{2}}\left(-\tau_{3}\right)^{A_{3}}$, we carry out a unitary transformation to new fields

$$
u_{i}^{\alpha a p}(y)=\frac{1}{4 \sqrt{2}} u_{i}^{\alpha a p}(y)=\frac{1}{4 \sqrt{2}} \sum_{A} \Gamma_{A}^{\alpha a p} \chi_{i}(A, y), \quad d_{i}^{\alpha a p}(y)=\frac{1}{4 \sqrt{2}} \sum_{A} B_{A}^{\alpha a p} \chi_{i}(A, y),
$$

where $y$ denotes a site on the "blocked" lattice with spacing $2 a$ and $A$ labels a site on a $2^{3}$ cube with its origin at site $y$. Each site $x$ of the original lattice corresponds, therefore, to a unique choice of $y$ and $A$. The combination

$$
q_{i}^{\alpha a p}(y)=\left(\begin{array}{c}
u_{i}^{\alpha}(y) \\
d_{i}^{\alpha}(y)
\end{array}\right)^{a p},
$$

may then be interpreted as a fermion field with spinor index $\alpha$, "taste" index $a$ and isospin index $p$.

We identify the pressure with the negative of the free energy density $-f=\left(T / V_{s}\right) \ln \mathcal{Z}$, where $V_{s}$ is the spatial volume of the lattice. In the large- $N_{f}$ limit fluctuations in the bosonic fields are ignored and the partition function is given by

$$
\begin{aligned}
\mathcal{Z} & =\int \mathrm{d} q \mathrm{~d} \bar{q} \exp \left(-\sum_{i=1}^{N} \sum_{k} \bar{q}_{i} M^{\dagger}(k) M(k) q_{i}\right) \exp \left(-S_{\mathrm{bos}}\right) \\
& =\left(\prod_{k} \operatorname{det} M(k)\right)^{2 N} e^{-S_{\mathrm{bos}}}
\end{aligned}
$$

where the fermionic action is written in terms of the fields $q$ and $\bar{q}$ recast in Fourier space. The effective fermion kinetic operator as a function of fermion momentum $k_{\mu}$ is 81

$$
\begin{aligned}
M(k)= & \sum_{\mu=1}^{3} \frac{i}{2}\left\{\left(\gamma_{\mu} \otimes \mathbb{1}_{2} \otimes \mathbb{1}_{2}\right) \sin 2 k_{\mu}+\left(\gamma_{4} \otimes \tau_{\mu}^{*} \otimes \mathbb{1}_{2}\right)\left(1-\cos 2 k_{\mu}\right)\right\} \\
& +\left(\mathbb{1}_{4} \otimes \mathbb{1}_{2} \otimes \mathbb{1}_{2}\right) \Sigma
\end{aligned}
$$

and the bosonic action is

$$
S_{\mathrm{bos}}=\frac{2 L_{s}^{2} L_{t} N \Sigma^{2}}{g^{2}}
$$

\footnotetext{
${ }^{1}$ We use the term "tastes" here to distinguish these 2 Kogut-Susskind flavors from our $N$ lattice and $N_{f}$ continuum flavors.
} 
In the tensor products in eq. (A-4) the first matrix acts on spinor, the second on "taste" and the third on isospinor indices. $\Sigma$ is the mean field equivalent of $\sigma(\tilde{x})$. Because the blocked lattice spacing is $2 a$, the Brillouin zone ranges from $-\pi / 2 a$ to $\pi / 2 a$, meaning that the allowed momenta are

$$
\begin{aligned}
& k_{\mu}=\frac{\pi n_{\mu}}{L_{s}} \quad n_{\mu}=0, \pm 1, \ldots, \pm L_{s} / 2 \text { for } \mu=1,2 \\
& k_{\mu}=\frac{\pi\left(2 n_{\mu}+1\right)}{2 L_{t}} n_{\mu}=0, \pm 1, \ldots, \pm L_{t} / 2 \text { for } \mu=3 \text {, }
\end{aligned}
$$

where $a$ has been set to 1 .

The determinant of eq. (A-4) is calculated as

$$
\operatorname{det} M(k)=\left[\frac{1}{4} \sum_{\mu=1}^{3}\left(\sin ^{2} 2 k_{\mu}+4 \sin ^{4} k_{\mu}\right)+\Sigma^{2}\right]^{8},
$$

so the unsubtracted free energy density is therefore

$$
f=-\frac{1}{L_{s}^{2} L_{t}} \ln \mathcal{Z}=\frac{-2 N}{L_{s}^{2} L_{t}} \sum_{k} \ln \operatorname{det} M(k)+\frac{2 N \Sigma^{2}}{g^{2}} .
$$

As was done with the lattice data, we normalise this by subtracting the zero temperature contribution so that the pressure as a function of temperature is defined as

$$
P=-\left(f_{T}-f_{0}\right)
$$

Finally, the Stefan-Boltzmann limit is found by calculating eq. ( $\mathrm{A}-9$ ) with $\Sigma \rightarrow 0$, which corresponds to $T \rightarrow \infty$.

\section{References}

[1] E. Babaev, Int. J. Mod. Phys. A16 (2001) 1175

[2] E. Babaev, Phys. Lett. B497 (2001) 323; E. Babaev, Phys. Rev. D 62 (2000) 074020.

[3] S.J. Hands, J.B. Kogut and C.G. Strouthos, Phys. Lett. B515 (2001) 407.

[4] N.D. Mermin and H. Wagner, Phys. Rev. Lett. 17 (1966) 1133;

S.R. Coleman, Commun. Math. Phys. 31 (1973) 259.

[5] J.B. Kogut, M.A. Stephanov and C.G. Strouthos, Phys. Rev. D58 (1998) 096001.

[6] E. Witten, Nucl. Phys. B145 (1978) 110.

[7] S.J. Hands and S.E. Morrison [UKQCD Collaboration], Phys. Rev. D59 (1999) 116002.

[8] S.J. Hands, A. Kocić and J.B. Kogut, Annals Phys. 224 (1993) 29. 
[9] G. Boyd, S. Gupta and F. Karsch, Nucl. Phys. B385 (1992) 481.

[10] H.A. Weldon, Phys. Rev. D40 (1989) 2410.

[11] J. Engels, J. Fingberg, F. Karsch, D. Miller and M. Weber, Phys. Lett. B252 (1990) 625.

[12] C. G. Strouthos, arXiv:hep-lat/0209143.

[13] S. Hands, B. Lucini and S. Morrison, Phys. Rev. D65 (2002) 036004.

[14] M. Kitazawa, T. Koide, T. Kunihiro and Y. Nemoto, Phys. Rev. D65 (2002) 091504.

[15] H. Kluberg-Stern, A. Morel, O. Napoly and B. Petersson, Nucl. Phys. B220 (1983) 447.

[16] C. Burden and A. N. Burkitt, Europhys. Lett. 3 (1987) 545. 